\title{
Structures, stresses, and fluctuations in the delayed failure of colloidal gels
}

\author{
Stefan Lindström, Thomas E Kodger, Joris Sprakel and David A Weitz
}

\section{Linköping University Post Print}

N.B.: When citing this work, cite the original article.

Original Publication:

Stefan Lindström, Thomas E Kodger, Joris Sprakel and David A Weitz, Structures, stresses, and fluctuations in the delayed failure of colloidal gels, 2012, Soft Matter, (8), 13, 3657-3664. http://dx.doi.org/10.1039/c2sm06723d

Copyright: Royal Society of Chemistry http://www.rsc.org/

Postprint available at: Linköping University Electronic Press http://urn.kb.se/resolve?urn=urn:nbn:se:liu:diva-76202 


\title{
Structures, stresses, and fluctuations in the delayed failure of colloidal gels
}

\author{
Stefan B. Lindström, ${ }^{* a b}$ Thomas E. Kodger, ${ }^{b}$ Joris Sprakel, ${ }^{b c}$ and David A. Weitz ${ }^{b}$
}

Sample-spanning networks of aggregated colloidal particles have a finite stiffness and deform elastically when subjected to a small shear stress. After some period of creep, these gels ultimately suffer catastrophic failure. This delayed yielding is governed by the association and dissociation dynamics of interparticle bonds and the strand structure of the gel. We derive a model which connects the kinetics of the colloids to the erosion of the strand structure and ultimately to macroscopic failure. Importantly, this model relates time-to-failure of the gel to an applied static stress. Model predictions are in quantitative agreement with experiments. It is predicted that the strand structure, characterized by its mesh size and strand coarseness, has a significant impact on delay time. Decreasing the mesh size or increasing the strand thickness makes colloidal gels more resilient to delayed yielding. The quench and flow history of gels modify their microstructures. Our experiments show that a slow quenching increases the delay time due to the coarsening of the strands; by contrast, preshear reduces the delay time, which we explain by the increased mesh size as a result of shear-induced fracture of strands.

\section{Introduction}

At sufficiently high volume fractions, colloids that interact through a short-range attraction form a gel, ${ }^{1,2}$ a percolated network structure with a finite, instantaneous yield stress. When a stress smaller than the yield stress is applied to the material, the initial response is elastic, but after some time the material can suddenly yield and start to flow. ${ }^{3,4}$ This phenomenon is known as delayed yielding or delayed failure. Colloidal gels are encountered in drug delivery systems ${ }^{5}$, food products ${ }^{6}$, cosmetics, paints, coatings and more, and the delayed yielding of these colloidal gels subject to gravitational forces, known as delayed collapse, limits the shelf-life of these products. Thus, their stability under loads is of direct interest and importance. Gravity induces compression in the bulk and shear near adhesive vertical walls. ${ }^{7,8}$ Under gravitational load as well as in simple shear, the time delay before yielding decreases exponentially with stress, ${ }^{3,9}$ indicating that a stress-dependent energy barrier governs failure at the particle-level. ${ }^{9}$ However, this picture is complicated by the strand architecture, which has an order-of-magnitude effect on delay time. ${ }^{10}$ To achieve predictive capability with a physical model, we must consider the full complexity of the system; this must include the effects of thermal fluctuations and of stresses at all levels of structure.

The creep response of colloidal gels typically displays three regimes: an initial elastic deformation, a subsequent primary creep regime where the creep rate decreases with strain and finally a catastrophic failure. ${ }^{3,4,10}$ This behavior is found in weak ${ }^{10}$ and strong colloidal gels ${ }^{3,4,10}$ as well as in polymeric gels ${ }^{11,12}$. Experiments indicate that the delay time $\tau$ decreases exponentially with applied shear stress $\sigma$ for strong gels of stearylated-silica particles in oil ${ }^{3}$ and carbon black particles in oil ${ }^{4}$. This suggests the presence of an energy barrier that

$0^{a}$ Mechanics, Department of Management and Engineering, the Institute of Technology, Linköping University, SE-58183 Linköping, Sweden. Fax: +46 13 149403; Tel: +46 13 281127; E-mail: stefan.lindstroem@gmail.com

$0^{b}$ School of Engineering and Applied Sciences, Harvard University, Cambridge, MA 02138, USA.

$0^{c}$ Laboratory of Physical Chemistry and Colloid Science, Wageningen University, Dreijenplein 6, $6703 \mathrm{HB}$, Wageningen, the Netherlands. decreases linearly with applied stress. ${ }^{4}$ A similar exponential scaling of delayed yielding time with stress is also observed for polymeric gels. ${ }^{11,12}$ Interestingly, if a preshear is applied to the colloidal gel, the delay time shows two distinct stress regimes. The delay time increases exponentially with decreasing stress, but with different exponential factors in the two regimes. This is observed for stearylated-silica particles in dodecane and carbon black in tetradecane, which both form strong gels, and in a weak polystyrene particle gel with a depletion attraction. ${ }^{10}$ The two regimes are attributed to the hierarchical architecture of particles and strands in conjunction with the stress-enhanced dissociation rate and association rate of colloid-colloid bonds. ${ }^{10}$

In this work, we validate and further develop the theory for delayed yielding in colloidal gels under a static loading. ${ }^{10} \mathrm{We}$ relate the applied stress, parameters of colloidal interactions and strand structure to the delayed yielding time. Our model emphasizes the importance of the gel strand structure, which can be modified by quench history ${ }^{13}$ and flow history ${ }^{14,15}$. We address how to control the strand structure to optimize material performance in delayed yielding by experimentally investigating the effects of preshear and quench rate on the delayed yielding time and interpret the results in light of our new model. This provides a crucial new understanding directly applicable to industrial processing of gel-based products.

\section{Yielding dynamics at multiple scales}

Colloidal gels have different structural features at clearly separable length-scales. At the microscale, the attractive particles aggregate to form gel strands, at the mesoscale, gel strands form a percolated network structure and at the macroscale the gel is a homogeneous material. These levels of structure are visible in a confocal microscope image of a colloidal gel comprising $1.1 \mu \mathrm{m}$ particles at a volume fraction $\phi=0.12$ in formamide/sulfolane depleted by $50 \mathrm{mg} / \mathrm{ml}, 500 \mathrm{kDa}$ dextran ${ }^{16}$ (Fig. 1a). It has been previously shown that these hierarchical structures strongly affect the moduli of gels, ${ }^{17}$ suggesting that they may also be pivotal to other mechanical properties 


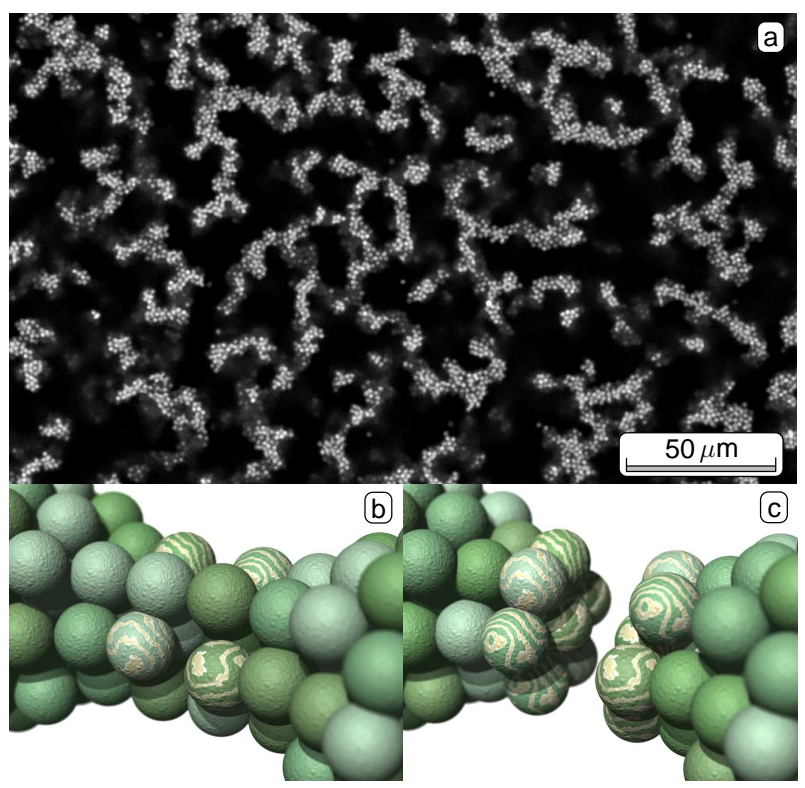

Fig. 1 (a) Representitive confocal microscope image of a colloidal gel of volume fraction $\phi=0.12$. Note the three levels of structure: particle-level, strand-level and homogeneous macroscopic level.

(b) Schematic illustration of two simultaneously detached colloid-colloid bonds (striped particles) at the neck of a strand. (c) Illustration of broken strand with seven broken bonds

including the delayed failure.

To describe the delayed yielding of these hierarchically structured materials, we model the system at multiple scales. A particle-level model describes the stress-enhanced bond dissociation. A strand-level model considers the collective dissociation/association dynamics of bonds at the neck of a gel strand and yields the strand dissociation rate. A macroscopic model relates the macroscopically applied stress to the strand dissociation rate and predicts the degradation of the material before damage localization and macroscopic failure.

\subsection{Particle-level model}

A colloidal particle of the formed gel is trapped in a potential well resulting from interactions with its neighbors. Within this potential well, irregular forces caused by thermal fluctuations of the surrounding medium induce particle vibrations, which can lead to the dissociation of a colloid-colloid bond. By considering the diffusion equation for the density distribution of particles in a colloid-colloid interaction potential, the dissociation rate of bonds can be derived. ${ }^{18}$ When no external force is applied across the bond, the dissociation rate becomes $k_{\mathrm{D}}=\omega_{0} \exp \left(-E_{\mathrm{A}} / k_{\mathrm{B}} T\right)$, where $\omega_{0}$ is the attempt frequency, $E_{\mathrm{A}}$ is the depth of the potential well, $k_{\mathrm{B}}$ is the Boltzmann constant and $T$ is the absolute temperature. The frequency $\omega_{0}$ depends on the diffusivity of the particles and the shape and depth of the potential barrier. ${ }^{18}$

When a force $f$ is applied across the weakly attractive bond, the energy barrier is lowered ${ }^{19}$ and the dissociation rate is modified, becoming ${ }^{20}$

$$
k_{\mathrm{D}}^{\prime}=k_{\mathrm{D}} \exp \left(\frac{f \delta}{k_{\mathrm{B}} T}\right), \quad f \delta<E_{\mathrm{A}},
$$

where $\delta$ is the characteristic width of the interaction potential. Here, the work performed by the applied force is approximated by $f \delta$. This model for the kinetics of bond dissociation has been experimentally verified for weakly attractive molecular systems. ${ }^{21}$ It should be mentioned that applying a force across the colloid-colloid bond slightly modifies $\omega_{0} \cdot{ }^{22}$ This effect is neglected in the present model.

\subsection{Strand-level model}

If the cross-section of a strand includes several particles, many dissociation events are required for a strand to break (Figs. 1bc). Due to thermal fluctuations, bonds also reform at an association rate $k_{\mathrm{A}}$, which is assumed to be independent of the applied stress. Since the thickness of a strand varies along its length, it is assumed that fracture will occur at an existing "neck", the weakest point along its contour, that has a crosssection of $n$ bonds. The integer number of intact bonds at the neck is denoted by $j \in[0, n]$.

During the rupture of a single strand, we assume that the macroscopic stresses are constant; which is achieved experimentally in so-called creep experiments. Even so, the force on the breaking strand may not be constant. When some of the bonds break at the neck of a straight strand, the tensile stiffness of the strand is significantly reduced, simply because the strand stiffness is the harmonic mean of the stiffness of its cross-sectional slices. When a straight strand weakens, the force it carries is redistributed to adjacent strands; as a result, the boundary conditions of the breaking strand is best described as a constant deformation. For a non-straight strand loaded in tension, elastic bending energy is distributed over the whole strand, not just its neck. Consequently, bonds breaking at the neck do not significantly change the apparent tensile stiffness of the strand; non-straight strands are thus subjected to constant force boundary conditions during fracture. Therefore, we explore two scenarios for strand rupture events: constant strand force $F$, or constant strand deformation, which corresponds to a constant bond force $f$.

We begin by examining the high stress limit, when $k_{\mathrm{D}}^{\prime} \gg$ $k_{\mathrm{A}}$, at constant deformation of the strand. In this case, association events are rare and negligible. The probability that a single bond is still intact at time $t$ after the application of a static load is $p_{1}(t)=\exp \left(-k_{\mathrm{D}}^{\prime} t\right)$. Then, the probability that a strand with a cross-section of $n$ particles is still intact at time $t$ becomes $p_{n}(t)=1-\left[1-p_{1}(t)\right]^{n}$. We integrate to find the average strand survival time $\tau_{\mathrm{D}}$, which yields the dissociation rate of the strands

$$
K_{\mathrm{D}}=\frac{1}{\tau_{\mathrm{D}}}=\left(-\int_{0}^{\infty} t^{\prime} \frac{\mathrm{d} p_{n}}{\mathrm{~d} t} \mathrm{~d} t^{\prime}\right)^{-1}=\frac{k_{\mathrm{D}}^{\prime}}{S_{n}} \approx \frac{k_{\mathrm{D}}^{\prime}}{\epsilon+\ln n} .
$$

Here, $S_{n}=\sum_{j=1}^{n} 1 / j$ and $\epsilon=0.5772 \ldots$ is Euler's constant. The last approximation of Eq. (2) holds for large values of $n$. 
Thus, at high applied loads the dissociation rate of a strand with many bonds in its cross-section is almost the same as the dissociation rate of the individual bonds that make up the strand.

If instead a constant force $F$ is applied to the strand, the force on each bond becomes $f=F / j$. When $j$ bonds remain, the time until the subsequent bond rupture is exponentially distributed with rate parameter $j k_{\mathrm{D}} \exp \left(F \delta / j k_{\mathrm{B}} T\right)$. The rate at which strands break can be estimated using the sum of expected times between each bond rupture

$$
K_{\mathrm{D}} \approx\left[\sum_{j=1}^{n} \frac{1}{j k_{\mathrm{D}}} \exp \left(-\frac{F \delta}{j k_{\mathrm{B}} T}\right)\right]^{-1}
$$

By substituting $x=j / n$ and assuming that $n$ is large, we obtain

$$
\begin{aligned}
K_{\mathrm{D}} & \approx k_{\mathrm{D}}\left[\sum_{j=1}^{n} \frac{1}{x} \exp \left(-\frac{F \delta}{n k_{\mathrm{B}} T} \cdot \frac{1}{x}\right) \frac{1}{n}\right]^{-1} \\
& \approx k_{\mathrm{D}}\left[\int_{0}^{1} \frac{1}{x} \exp \left(-\frac{F \delta}{n k_{\mathrm{B}} T} \cdot \frac{1}{x}\right) \mathrm{d} x\right]^{-1} \\
& =\frac{k_{\mathrm{D}}}{E_{1}\left(F \delta / n k_{\mathrm{B}} T\right)} \\
& \approx \frac{1}{\ln \left(1+n k_{\mathrm{B}} T / F \delta\right)} \cdot k_{\mathrm{D}} \exp \left(\frac{F \delta}{n k_{\mathrm{B}} T}\right) .
\end{aligned}
$$

In the final approximation, the exponential integral $E_{1}(z)=$ $\int_{1}^{\infty} t^{-1} e^{-z t} \mathrm{~d} t$ is approximated using elementary functions for convenience. ${ }^{23}$ According to Eq. (4), the strand dissociation rate for constant strand force is much increased as compared to constant strand deformation in Eq. (2).

In the low stress limit, when $k_{\mathrm{D}}^{\prime} \ll k_{\mathrm{A}}$, bond dissociation events are followed almost immediately by an association event. Consequently, all bonds of the cross-section must break within an $\mathcal{O}\left(1 / k_{\mathrm{A}}\right)$ time for a strand to break, thus making strand rupture a much more rare and cooperative event. We use the following thought experiment to derive the dissociation rate of strands: Assume that the fracture gap does not widen when the strand breaks. Then, the probability that one particular bond of the cross-section is broken at a given time is $P_{1}=k_{\mathrm{D}}^{\prime} / k_{\mathrm{A}}$. The probability that all bonds are broken is $P_{1}^{n}$. That is, $P_{1}^{n}$ is the fraction of time that the strand spends in the broken state. At the point of strand fracture, the expected time before one of the bonds reforms and restores the strand is $1 / n k_{\mathrm{A}}$. Thus, $1 / n k_{\mathrm{A}}$ is the typical duration of each fracture. This yields the dissociation rate of strands,

$$
K_{\mathrm{D}} \approx n k_{\mathrm{A}} P_{1}^{n}=n k_{\mathrm{A}}\left(\frac{k_{\mathrm{D}}}{k_{\mathrm{A}}}\right)^{n} \cdot \exp \left(\frac{n f \delta}{k_{\mathrm{B}} T}\right) .
$$

Now, suppose the gap is allowed to widen as a strand breaks. This precludes the re-association of bonds after the strand fracture event. However, for an intact strand, the dissociation rate cannot depend on the future fate of the strand after fracture due to the laws of causality. Hence, the strand dissociation rate must still be given by Eq. (5) when strand fracture is irreversible.

\subsection{Macroscopic model}

At the macroscale, we take a mean-field approach. When a small stress is applied to a statistically homogeneous material, the initial microscopic strand fractures are distributed over the volume of the sample. ${ }^{24,25}$ We assume that the degradation proceeds in this dispersed failure mode, preserving spatial homogeneity. Eventually, there is damage localization, which means that cracks much larger than the mesh size of the pristine material form. This finally leads to critical fracture and macroscopic failure of the material. We propose that the delay time is well estimated by the time-scale of the initial static fatigue, occurring prior to the final macroscopic failure, while the duration of critical crack propagation, which is much more rapid, can be neglected.

Consider a strand structure of characteristic mesh size $\xi$. The area density of strands in a hypothetical yield surface is $\rho \sim 1 / \xi^{2}$. From a mean-field, equal load-sharing approximation, the tensile and transverse shear force components on a strand become

$$
\begin{aligned}
\bar{F}_{\|}(\hat{\boldsymbol{p}}) & =(\boldsymbol{\sigma} \cdot \hat{\boldsymbol{p}}) \cdot \hat{\boldsymbol{p}} / \rho(\hat{\boldsymbol{p}}) \\
\bar{F}_{\perp}(\hat{\boldsymbol{p}}) & =|(\boldsymbol{\delta}-\hat{\boldsymbol{p}} \hat{\boldsymbol{p}}) \cdot(\boldsymbol{\sigma} \cdot \hat{\boldsymbol{p}})| / \rho(\hat{\boldsymbol{p}})
\end{aligned}
$$

respectively. Here, overbars are used to denote spatial average, $\sigma$ is the stress tensor, $\delta$ is the unit tensor and $\hat{p}$ is the unit vector in the axial direction of the strand. The axial force $\bar{F}_{\|}$ modifies the bond energy barrier as previously described. We assume that a finite shear force $\bar{F} \perp$ across the strand also leads to enhanced dissociation, lowering the energy barrier of each bond by a factor $\alpha \bar{F}_{\perp} \delta / j k_{\mathrm{B}} T$, where $\alpha>0$ is a constant factor. The two components $\bar{F}_{\|}$and $\bar{F}_{\perp}$ both contribute to the effective strand force $\bar{F}$ controlling the strand dissociation rate. As a first-order approximation, we make the linearization $\bar{F}=\bar{F}_{\|}+\alpha \bar{F}_{\perp}$. The strands with highest dissociation rate are oriented in the direction which maximizes $\bar{F}(\hat{\boldsymbol{p}})$. In a simple shear geometry with an applied shear stress $\sigma_{12}$, we show in Appendix A that the effective strand force can be written as $\bar{F}=\sigma / \rho$, where $\sigma \sim \sigma_{12}$. For the constant strand force assumption, we thus have $F=\bar{F}=\sigma / \rho$, while for the constant strand deformation assumption, we have $f=\bar{F} / n=$ $\sigma / n \rho$.

Strands break with a dissociation rate $K_{\mathrm{D}}$ and re-form with an association rate $K_{\mathrm{A}}$. This dissociation/association dynamics leads to a birth-death type ordinary differential equation (ODE) for $\rho=\rho(\hat{\boldsymbol{p}}, t)$. We drop the direction-dependence of $\rho$ for brevity, understanding that we study the most probable fracture plane:

$$
\frac{\mathrm{d} \rho}{\mathrm{d} t}=-K_{\mathrm{D}} \rho+K_{\mathrm{A}}\left(\rho_{0}-\rho\right), \quad \rho(0)=\rho_{0},
$$

where $\rho_{0}$ is the initial area density of strands. Here, $K_{\mathrm{D}}$ is a function of $\rho$ through the effective bond force $\bar{F}$. As $\rho$ diminishes, the material becomes weaker, $\bar{F}$ increases and the strand dissociation accelerates.

We take the characteristic delay time $\tau$ to be the time required for $\rho$ to decrease from $\rho_{0}$ to $\rho_{0} / e$. It is straight-forward 
to compute $\tau$ by solving Eq. (7) numerically. The predicted behavior is, however, not directly apparent from the ODE.

For the constant strand deformation assumption and negligible association rate of strands, $K_{\mathrm{A}}=0$, it is possible to obtain an analytical estimate for $\tau$. We have

$$
\frac{\mathrm{d} \widetilde{\rho}}{\mathrm{d} \widetilde{t}}=-\widetilde{\rho} \exp \left[\widetilde{n} C \sigma\left(\frac{1}{\widetilde{\rho}}-1\right)\right], \quad \widetilde{\rho}(0)=1,
$$

where $\widetilde{\rho}=\rho / \rho_{0}$ is nondimensional area density, $\tilde{t}=\left.t K_{\mathrm{D}}\right|_{t=0}$ is nondimensional time, $C=\delta / n \rho_{0} k_{\mathrm{B}} T$ has the unit of compliance and

$$
\tilde{n}=\left\{\begin{array}{cc}
n, & k_{\mathrm{D}}^{\prime} \ll k_{\mathrm{A}} \\
1, & k_{\mathrm{D}}^{\prime} \gg k_{\mathrm{A}} .
\end{array}\right.
$$

When $\tilde{n} C \sigma \gtrsim 1$, which according to our experiments is the case for strong gels, numerical solutions of Eq. (8) show that the nondimensional delay time is $\widetilde{\tau} \approx 1 / \widetilde{n} C \sigma$. Returning to dimensional form, we arrive at our main result

$$
\tau \approx \begin{cases}\frac{1}{n^{2} k_{\mathrm{A}}}\left(\frac{k_{\mathrm{A}}}{k_{\mathrm{D}}}\right)^{n} \cdot \frac{1}{C \sigma} e^{-n C \sigma}, & k_{\mathrm{D}}^{\prime} \ll k_{\mathrm{A}} \\ \frac{S_{n}}{k_{\mathrm{D}}} \cdot \frac{1}{C \sigma} e^{-C \sigma} & , \quad k_{\mathrm{D}}^{\prime} \gg k_{\mathrm{A}} .\end{cases}
$$

Equation (10) predicts two stress regimes separated by a critical stress $\sigma_{\mathrm{c}}$. Solving $k_{\mathrm{A}}=k_{\mathrm{D}}^{\prime}$ for $\sigma_{\mathrm{c}}$ yields

$$
\sigma_{\mathrm{c}}=\frac{1}{C} \cdot \ln \frac{k_{\mathrm{A}}}{k_{\mathrm{D}}} .
$$

By virtue of the analytical solution (10), it is immediately obvious that two stress regimes of different exponential scaling should be seen in the delay time, as previously observed in experiments. ${ }^{10}$

\section{Model validation}

\subsection{Materials and methods}

Experiments are conducted on three different systems: a thermoreversible stearylated-silica particle gel $\left(\mathrm{SiO}_{2}\right)$, a carbon black gel (CB) and a polystyrene particle gel (PS). The first two materials form strong gels, with attraction strengths of several tens to hundreds of $k_{\mathrm{B}} T$, while the latter depletioninduced gelation is weak with attraction strengths of order $10 k_{\mathrm{B}} T$. Gel conditions are chosen such that no visible sedimentation or syneresis is observed in the gel state over at least one week after preparation or pre-shear rejuvenation, thus preventing our experiments from being influenced by such secondary effects.

The $\mathrm{SiO}_{2}$ system is prepared from $160 \mathrm{~nm}$ diameter stearylated-silica particles in dodecane at different volume fractions $\phi=\{0.20,0.25,0.33\}$. These systems are fluid and well-dispersed at high temperatures, but undergo a gel transition around $29^{\circ} \mathrm{C}$. The sample is, in each experiment, loaded into a concentric cylinder geometry in a stress-controlled rheometer (MCR501, Anton Paar) and melted at $40^{\circ} \mathrm{C}$. The temperature is subsequently lowered to $5^{\circ} \mathrm{C}$ and the suspension is allowed to gel for $30 \mathrm{~min}$. The gel is then presheared with varying total strain $\gamma_{\text {pre }}$ at a constant shear rate $\dot{\gamma}=$ $0.01 \mathrm{~s}^{-1}$. After this preshear treatment, the sample is left to equilibrate for an additional $15 \mathrm{~min}$. For the CB system, carbon black particles are suspended in tetradecane at $8 \mathrm{wt} \%$. The PS system consists of $480 \mathrm{~nm}$ diameter polystyrene particles at $\phi=0.30$ in water with dextran added to induce depletion interactions to form the gel. Before each measurement, we subject these two systems to a strong preshear $\dot{\gamma}=500 \mathrm{~s}^{-1}$ for $60 \mathrm{~s}$, and let them equilibrate quiescently for $15 \mathrm{~min}$.

Direct imaging of gel structure with $\gamma_{\text {pre }}>0$ is achieved by combining a spinning-disk confocal (Yokogawa CSU22) with a commercial rheometer (Malvern, HR Nano). A custommade Couette geometry allows for the observation of the shear-gradient plane across the entire gap. Gels are subjected to a strong preshear $\dot{\gamma}=200 \mathrm{~s}^{-1}$ for $60 \mathrm{~s}$ with an equilibration time of $5 \mathrm{~min}$, or until the gel visually does not undergo structural rearrangement in the observed field of view, $\sim 100 \mu \mathrm{m}$. The sample is then strained continuously at a shear rate of $\dot{\gamma}=0.01 \mathrm{~s}^{-1}$; images are captured throughout up to $\gamma_{\text {pre }}=3$.

\subsection{Comparison with experiments}

The creep response of colloidal gels usually displays three regimes: an initial elastic deformation, a primary creep regime where the creep rate decreases with strain and finally a catastrophic failure. This is exemplified by the creep curves of the $\mathrm{SiO}_{2}$ system with $\phi=0.25$ for stresses in the range $\sigma \in$ [6.3, 12] Pa (Fig. 2a). The slowly accelerating deformation in the secondary creep regime, which eventually develops into the catastrophic failure, is in accord with our picture of slow material degradation through stochastic strand-rupture events, followed by damage localization and rapid growth of macroscopic cracks. We take the delayed yielding time to be the time $\tau$ at which the shear rate is maximized. Each creep measurement thus renders a single $\tau$ for one $\sigma$; several repeats are performed for each $\sigma$ to yield $\tau(\sigma)$.

When $\tau(\sigma)$ is plotted for the $\mathrm{SiO}_{2}$ system with $\gamma_{\text {pre }}=0$, we observe a single exponential regime. However, for a finite preshear $\gamma_{\text {pre }}=3$, two exponential regimes appear (Fig. 2b). This is exactly the behavior predicted by our model. For the presheared gel, we observe the two stress regimes of Eq. (10), corresponding to strand dissociation rates given by Eqs. (2) and (5), respectively. For the gel without flow history, only the low stress behavior can be observed, simply because the yielding in the high stress regime, Eq. (4), is too fast to manifest as delayed yielding.

To demonstrate the generality of our approach, we measure $\tau(\sigma)$ for the two different gel systems, the weak PS gel and the strong $\mathrm{CB}$ gel with preshear. Both exhibit two stress regimes in $\tau$, in agreement with the model prediction (Figs. 2cd). Without flow history, carbon black gels exhibit only one stress regime ${ }^{4}$. It is worth noticing that the delay time appears to diverge to infinity for the two unsheared strong gels, $\mathrm{SiO}_{2}$ and $\mathrm{CB}$ (Figs. 2bd), suggesting that reassociation of entire strands may become important at very low stresses.

The four unknown parameters, $k_{\mathrm{D}}, k_{\mathrm{A}}, n$ and $C$ can be 

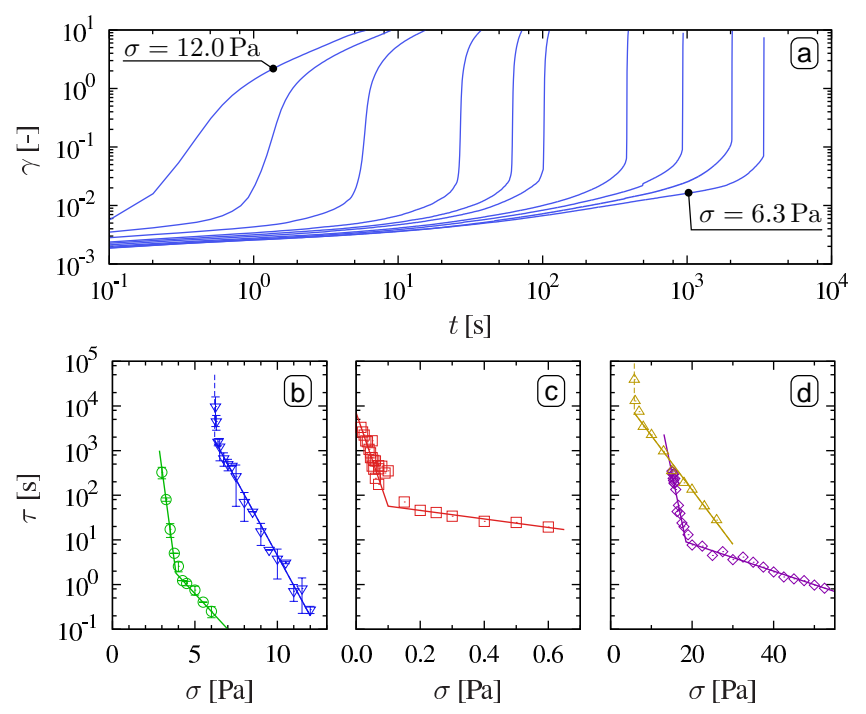

Fig. 2 (a) Creep response for different shear stresses $\sigma=\{12,11,10,9,8,7,6.75,6.5,6.3\}$ Pa for $\mathrm{SiO}_{2}$ gels with $\phi=0.25$. (b) Time delay $\tau$ as a function of shear stress $\sigma$ for $\mathrm{SiO}_{2}$ gels with $\phi=0.25, \gamma_{\text {pre }}=0$ (triangles) and $\gamma_{\text {pre }}=3$ (circles). (c) Delay time vs shear stress for weak PS gels. (d) Delay time vs shear stress for strong carbon black gels: CB system (diamonds) and $6 \mathrm{wt} \%$ carbon black in mineral oil with no preshear ${ }^{4}$ (triangles). The solid lines in plots $\mathrm{b}-\mathrm{d}$ indicate one or two exponential relations. The dashed lines indicate apparent diversion to infinity.

obtained directly from the slopes and intercepts of the two regimes. ${ }^{10}$ Alternatively, they can be obtained from a fitting procedure using numerical solutions of Eq. (7). The latter method is employed in this work for the PS, CB and $\mathrm{SiO}_{2}$ systems (solid lines of Figs. 2bcd). The extracted parameters are compiled in Table 1, in which the data are sorted in ascending order of gel strength. Interestingly, both structural and dynamic parameters that characterize the gels can be obtained from a single type of rheology experiment. As the depth of the interaction potential increases, going from weak to stronger gels in Table 1, the typical elastic energy $W=\sigma_{\mathrm{c}} C$ required to initiate erosion increases. Here, $W$ is normalized by $k_{\mathrm{B}} T$.

Although the critical stress $\sigma_{\mathrm{c}}=\ln \left(k_{\mathrm{A}} / k_{\mathrm{D}}\right) / C$ separating the two stress regimes is derived as an order-of-magnitude

Table 1 Structural parameters for various gel systems obtained from delayed yielding experiments and Eq. (7): strand coarseness $n$, bond "compliance" $C$ and quiescent bond dissociation and association rates $k_{\mathrm{D}}$ and $k_{\mathrm{A}}$. Here, $\sigma_{\mathrm{c}}$ is the computed critical stress separating the stress regimes and $W$ is the typical elastic energy at break, normalized by $k_{\mathrm{B}} T$.

\begin{tabular}{lcccccccc}
\hline & $\phi$ & $\gamma_{\text {pre }}$ & $n$ & $C$ & $k_{\mathrm{D}}$ & $k_{\mathrm{A}}$ & $\sigma_{\mathrm{c}}$ & $W$ \\
& {$[\%]$} & {$[-]$} & {$[-]$} & {$\left[\mathrm{Pa}^{-1}\right]$} & {$\left[\mathrm{s}^{-1}\right]$} & {$\left[\mathrm{s}^{-1}\right]$} & {$[\mathrm{Pa}]$} & {$[-]$} \\
\hline $\mathrm{PS}$ & 30 & 3000 & 17 & 1.5 & 0.045 & 0.08 & 0.38 & 0.2 \\
$\mathrm{CB}$ & $8 \mathrm{wt}$ & 3000 & 25 & 0.045 & 0.26 & 0.75 & 24 & 0.7 \\
$\mathrm{SiO}_{2}$ & 25 & 0 & 4.5 & 0.33 & 0.0070 & 0.60 & 13.5 & 4.5 \\
$\mathrm{SiO}_{2}$ & 25 & 3 & 5.5 & 0.90 & 0.0070 & 0.60 & 4.9 & 4.5 \\
\hline
\end{tabular}
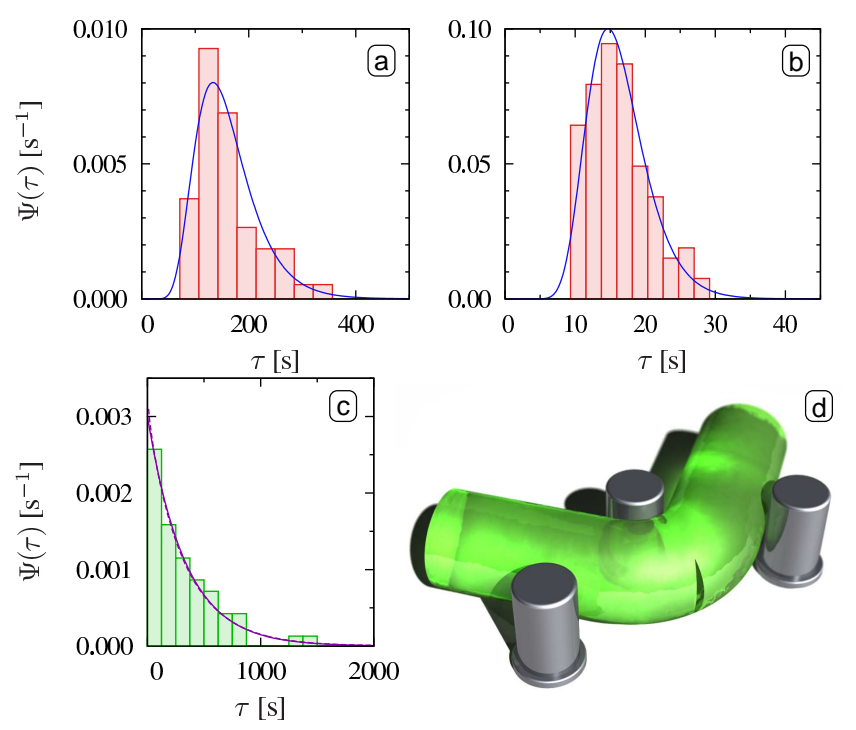

Fig. 3 Probability densities normalized to unity for the delay time of $\mathrm{SiO}_{2}$ gels with $\phi=0.25$ at two different shear stresses (a) $\gamma_{\text {pre }}=0, \sigma=8 \mathrm{~Pa}$ and (b) $\gamma_{\text {pre }}=1.2, \sigma=4 \mathrm{~Pa}$. Solid lines are fitted log-normal distributions. (c) Normalized probability density of delay time for a polymer gel in a three-point bending geometry. ${ }^{11}$ Solid line is a fitted exponential distribution and dashed line (overlapping) is the Weibull distribution. (d) Failure in a three-point bending geometry.

estimate, there is quantitative agreement with experimentally observed critical stresses of strong gels: In the CB system, critical stress is computed to be $\sigma_{\mathrm{c}}=24 \mathrm{~Pa}$, while the experimental value is $\sigma_{\mathrm{c}}=20 \mathrm{~Pa}$ (Fig. 2d). Equally good agreement is seen for the presheared $\mathrm{SiO}_{2}$ systems.

According to our model, yielding is the result of a large number of stochastic events. Damage localization does not occur until there is a sufficiently large number of ruptured strands. For this equal load-sharing, dispersed failure mode, the macroscopic material lifetimes are expected to be lognormally distributed for systems of finite size. ${ }^{25}$ To verify if our material obeys these log-normal statistics we perform 150 experiments on the $\mathrm{SiO}_{2}$ system with $\phi=0.25$ with and without preshear to obtain the normalized probability density of delay times $\Psi(\tau)$. The delay times between individual runs are uncorrelated, asserting that our pre-treatment generates a history-independent initial condition. The resulting distributions are shown in Figs. 3ab. The log-normal distribution (solid lines) provides an excellent fit and captures the prominent skewness and tails of the measured distributions. This further strengthens our argument that thermally driven stochastic events are responsible for delayed yielding.

For a soft solid in a three-point bending geometry, delay times are observed to be exponentially distributed (Fig. 3c). ${ }^{11}$ This is not in contradiction to our result; our investigation considers the behavior in a region of constant macroscopic stresses, whereas the three-point bending geometry induces a stress concentration on the surface of the sample at the point of greatest curvature (Fig. 3d). In that case, the observed exponential distribution can be explained by two possible mech- 


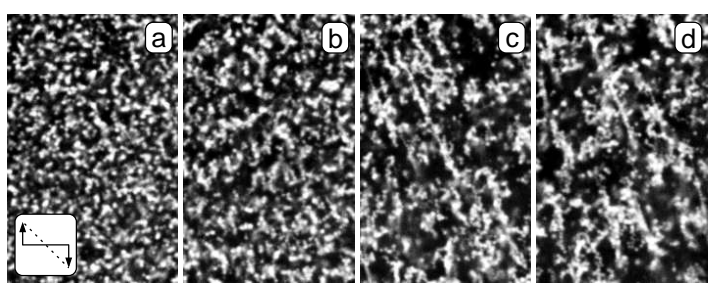

Fig. 4 Confocal microscope images of an index and denity matched colloidal gel for different amounts of preshear $\gamma_{\text {pre }} \in\{0,1,2,3\}$. The shear-gradinet plane is imaged with upward flow direction. The volume fraction is in the lower range, $\phi=0.15$, to enhance visibility.

anisms. One possibility is that a single stochastic event is required for fracture, implying that macroscopic failure is a Poisson process. Alternatively, the material breaks at its weakest point in the small region of the stress concentration, which typically produces the Weibull distribution ${ }^{26}$ reminiscent of an exponential distribution.

\section{Processing conditions and delay time}

One method of making gels more resilient to delayed yielding is to increase the ratio between association and dissociation rates, $k_{\mathrm{A}} / k_{\mathrm{D}}$, of individual bonds, which promotes the stability at low stresses, as predicted by Eq. (10). This would require changes in the chemistry of the system. For products such as cosmetics or food, changing chemistry often has adverse effects on other critical product properties. However, this model also predicts a significant effect of the mesoscale structure on the delayed yielding time. This structure can be modified for instance by changing volume fraction ${ }^{13}$, applying a preshear ${ }^{14,27}$ or by changing the quench history, for instance by allowing the strands to coarsen at a shallow quench before making the gel thermally stable at a deeper quench. ${ }^{13}$ We experimentally investigate how the application of preshear and different quench rates affects the delayed yielding and interpret the results through our proposed model in terms of changes in the gel structure.

\subsection{Effects of preshear}

We prepare a gel system optimized for imaging to study the development of the strand structure when sheared at a constant rate, which is known to induce aggregation of particle clusters in attractive ${ }^{14}$ as well as stabilized ${ }^{28}$ colloidal systems. A colloidal gel is formed in a suspension of index and density matched particles, made attractive by addition of a linear polymer which induces a depletion attraction between the particles. ${ }^{16}$ After formation, a confocal microscopy image shows that the gel has a finely stranded structure (Fig. 4a) characteristic of irreversible gelation. Upon applying a constant shear rate, $\dot{\gamma}=0.01 \mathrm{~s}^{-1}$, we see the gel evolving in time in the sequence of images (Fig. 4bcd); the initially finely-stranded gel becomes gradually more coarse and heterogeneous, due to disproportionation of the gel structure. When the applied
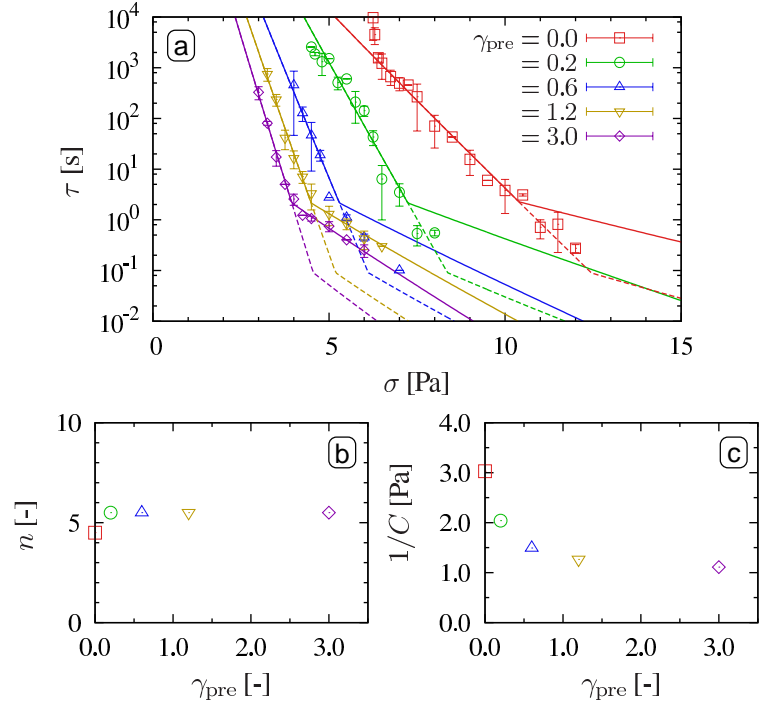

Fig. 5 (a) The delay time $\tau$ as a function shear stress $\sigma$ in simple shear for a stearylated-silica gels subjected to different amounts of

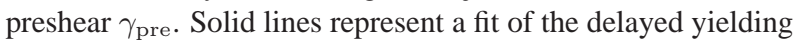
model using the constant strand deformation assumption, while dashed lines represent the constant strand force assumption.

(b) Bonds per strand cross-section $n$ from fitting procedure. (c) The inverse $1 / C$ of the compliance fitting parameter. Note that $1 / n C$ is proportional to the area density of strands $\rho_{0}$.

preshear exceeds a total strain of approximately $\gamma_{\text {pre }}=1$, anisotropy develops in the structure. The coarsening of the structure leads to decreasing strand area density $\rho_{0}$ and thus an increase of the compliance parameter $C$ with preshear. The coarsening of the structure should also increases $n$. This qualitative picture is confirmed in our investigation (Table $1, \mathrm{SiO}_{2}$ ). To quantify the effects of preshear in more detail, we use the $\mathrm{SiO}_{2}$ system with $\phi=0.25$ and measure the delay time. We plot the delay time as a function of shear stress for various preshears in the range $\gamma_{\text {pre }} \in[0,3]$. Each data point is the average of at least three measurements. All sets of data are fit by solving Eq. (7) numerically, using the strand dissociation rate $K_{\mathrm{D}}$ given by Eqs. (2), (4) and (5), respectively. Remarkably, the whole family of curves can be fit using the same values for $k_{\mathrm{D}}=7.0 \cdot 10^{-3} \mathrm{~s}^{-1}$ and $k_{\mathrm{A}}=0.60 \mathrm{~s}^{-1}$, as shown in Fig. 5. This is consistent with the idea that dissociation and association rates originate from chemistry and are unaffected by structure. Only $C$ and $n$ must be fit for each individual curve since they change with structure (Figs. 5bc).

When no preshear is applied to the gel, one stress regime is observed in which the delay time grows exponentially with decreasing stress. At a limiting stress of about $6.3 \mathrm{~Pa}$, the delay time diverges to very large values, as is also reported for unsheared carbon black gels. ${ }^{4}$ When preshear is applied, the delay times begin to exhibit two stress regimes with different exponential rates, as predicted by Eq. (10). Our interpretation is that in the unsheared case, the gel strands are non-straight. The strands are thus subjected to constant force boundary conditions and should follow the dashed lines of Fig. 5a. Sufficient preshear pulls out the slack of the structures, producing 


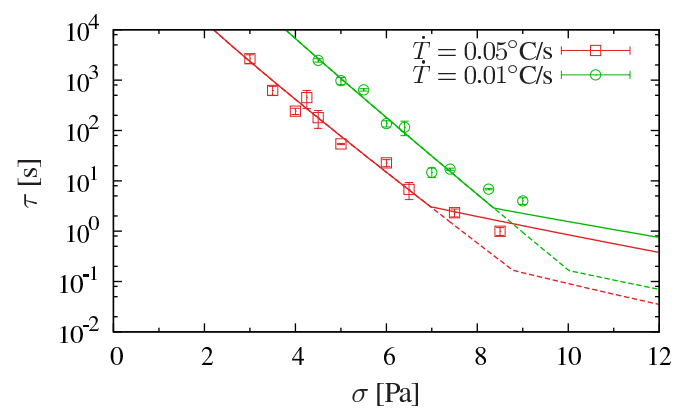

Fig. 6 The delay time $\tau$ as a function shear stress $\sigma$ in simple shear for the $\mathrm{SiO}_{2}$ gel quenched at different temperature rates $\dot{T}$. Solid lines represent a fit of the delayed yielding model using the constant strand deformation assumption, while dashed lines represent the constant strand force assumption.

straighter strands. With increasing amounts of preshear, we thus see a transition to constant strand deformation conditions, so that the delay time follows the solid lines of Fig. 5a.

The typical number of bonds $n$ at the strand necks is obtained from the model fit (Fig. 5b). It is slightly increasing from 4.5 to 5.5 when preshear is applied indicating that the number of bonds at the necks of the strands is not much affected by shear. Although aggregates coalesce to form a coarser structure when sheared (Fig. 4), the critical weak links of the structure do not strengthen significantly. The inverse compliance $1 / C$ decreases with preshear (Fig. 5c) because of the increasing mesh size (Fig. 4). Preshear has an adverse effect on the stability of these colloidal gels with respect to yielding. Clearly, flow history has a great impact on the performance of these materials.

\subsection{Effects of quench history}

The $\mathrm{SiO}_{2}$ system $(\phi=0.25)$ is prepared as described in the previous Sec. 4.1. Note that the particles used in the experiments of this section are from a different batch, precluding a quantitative comparison with data from previous sections. No preshear is applied and a different quenching procedure is used: The gel is melted at $40^{\circ} \mathrm{C}$ and the temperature reduced to $5^{\circ} \mathrm{C}$ using different temperature ramp rates $\dot{T} \in[0.01,0.05]^{\circ} \mathrm{C} / \mathrm{s}$. The sample is then left to equilibrate for $5 \mathrm{~min}$ before each delayed yielding experiment. The order between fast and slow quench rate experiments is random to eliminate any bias.

We investigate the delay time as a function of shear stress for two different quench rates: $0.01^{\circ} \mathrm{C} / \mathrm{s}$ and $0.05^{\circ} \mathrm{C} / \mathrm{s}$. Only one exponential stress regime is observed in both cases, perhaps with a slight indication of second regime at high stresses, as shown in Fig. 6. A slower quench rate makes the gel more resistant to yielding; at each stress, the delay time differs by an order of magnitude between the experiments.

The two data sets are fit by solving Eq. (7) numerically, with $K_{\mathrm{D}}$ given by Eqs. (2), (4) and (5), respectively. Because the microstructures of the rapidly quenched gel should be similar to those of the unsheared gel in Sec. 3.2, the val- ues of $C=0.33 \mathrm{~Pa}^{-1}, n=4.5$ and $k_{\mathrm{A}}=0.60 \mathrm{~s}^{-1}$ are taken from Table 1 . The dissociation rate is the only fitting parameter for the rapidly quenched gel: $k_{\mathrm{D}}=2.2 \cdot 10^{-1} \mathrm{~s}^{-1}$, which is a factor three greater than for the previous batch of particles (Table 1). At the slower quench rate, the gel microstructure is modified. The slow quench data are thus fit using the structure parameters: $C=0.29 \mathrm{~Pa}^{-1}$ and $n=5.5$. There is a $22 \%$ increase in $n$, which is indicative of a coarsening of the structure. At the same time, there is a $7 \%$ decrease in $\rho_{0} \propto 1 / n C$ showing that the strand density is slightly reduced by a slower quench rate.

\section{Conclusions}

The delayed yielding of colloidal gels is strongly affected by their hierarchical structure. In the limit of small stresses, failure of individual strands is serendipitous; several bond dissociation events must occur almost simultaneously for a strand to break. An expression for the delay time is derived from the association/dissociation dynamics of colloid bonds, the strand coarseness and mesh size.

After quenching, the gel structure is spinodal and finely stranded. In this case, the delayed yielding time decreases exponentially with increasing stress. In the low stress limit, it is predicted in Eq. (10) that coarse-stranded gels are stronger from the point of delayed yielding. If a gel is allowed to coarsen by maintaining it at a shallow quench for some time, and then is stabilized by a deeper quench, this coarser structure will perform better in delayed yielding, which is also observed experimentally.

When a preshear is applied to the gel, slack is pulled out of its structures so that strands become straighter. At sufficient amounts of preshear $\gamma \gtrsim 1$, two distinct stress regimes appear, where delay time increases exponentially with decreasing stress, but with different exponential factors in the two regimes. Some of the gel strands break during preshear and the dissociation rate is further enhanced as the force on the remaining strands increases. Consequently, preshear reduces the delay time.

To produce a gel which performs optimally in delayed yielding, it should be slowly quenched, while avoiding excessive shearing. In the industrial processing of gels, adverse effects of preshear highlight the importance of flow geometry design, while the dependence on quench rate suggests that production speed could be optimized through engineering controls to enhance performance by limiting delayed yielding. The model presented here provides guidance for this optimization.

Although the delayed yielding in simple shear is not directly shown to govern gravity-induced delayed collapse, we emphasize this possibility, particularly for strong gels, which are otherwise thermally stable under quiescent conditions. It is clear from the explicit relation between the general stress tensor and the strand dissociation rate, that the proposed erosion mechanism is present in both shear and compression (Appendix A). Indeed, gravity induces both compressive stresses in the bulk 
of the gel and shear stresses near adhesive vertical walls of its container, ${ }^{7,8}$ either of which could cause delayed collapse.

Delayed yielding and the difficulties in its prediction is a problem for a wide range of heterogeneous solids, including steel ${ }^{29}$, wood $^{30}$, ceramics ${ }^{31}$ and soft solids ${ }^{9,11}$. Although delayed yielding in these materials have somewhat different physical origin on the microscopic level, it originates from the interplay between thermal fluctuations, structure, applied stresses and sometimes corrosion phenomena. Colloidal gels, by virtue of their clear separation of structural length-scales, can be regarded as simple model systems for studying these common yielding mechanisms of heterogeneous solids. Our results thus provide insight into the fracture of hierarchically structured materials under a constant loading.

\section{Acknowledgements}

S.B.L. thanks the Alf de Ruvo Memorial Foundation of SCA AB and J.S. thanks the Netherlands Organization for Scientific Research (NWO) for financial support. This work is supported by the Harvard MRSEC (DMR-0820484) and NSF (DMR-1006546).

\section{A Stress state and strand forces}

A strand with its length axis in the $\hat{\boldsymbol{p}}$ direction is subjected to tensile and transverse shear force components, $\bar{F}_{\|}(\hat{\boldsymbol{p}})$ and $\bar{F}_{\perp}(\hat{\boldsymbol{p}})$ according to Eqs. (6a) and (6b). The strands of highest dissociation rate are oriented in the direction $\hat{\boldsymbol{q}}$ which maximizes the effective strand force $\bar{F}=\bar{F}_{\|}+\alpha \bar{F}_{\perp}$.

In simple shear, the non-zero components of the stress tensor $\boldsymbol{\sigma}$ are $\sigma_{12}=\sigma_{21}$. From symmetry, we find that $\hat{\boldsymbol{q}}=$ $[\cos \varphi, \sin \varphi, 0],-\pi / 2<\varphi<\pi / 2$. Maximizing $\bar{F}=$ $\sigma_{12}(\sin 2 \varphi+\alpha|\cos 2 \varphi|) / \rho$ renders $\varphi=\frac{\pi}{4} \pm \frac{1}{2} \arctan \alpha$. From symmetry, it is expected that fracture planes develop for $\varphi=\frac{\pi}{4}$.

In compression, the only non-zero stress component is $\sigma_{22}<0$. We consider fracture planes with normal $\hat{\boldsymbol{q}}=$ $[\cos \theta, \sin \theta, 0],-\pi / 2<\theta<\pi / 2$, recognizing that there is an axial symmetry around the 2-direction. Maximizing $\bar{F}=$ $\sigma_{22}\left(\sin ^{2} \theta-\frac{1}{2} \alpha|\sin 2 \theta|\right) / \rho$ gives $\theta= \pm \frac{1}{2} \arctan \alpha$. Other load cases can be treated in a similar manner.

In general, the effective strand force in the fracture plane can be written as $\bar{F}=\sigma / \rho$, where $\sigma \sim \sigma_{12}$ in simple shear, which is our main concern in this work.

\section{References}

1 V. Prasad, V. Trappe, A. D. Dinsmore, P. N. Segre, L. Cipelletti and D. A. Weitz, Faraday Discuss., 2003, 123, 1-12.

2 P. J. Lu, E. Zaccarelli, F. Ciulla, A. B. Schofield, F. Sciortino and D. A. Weitz, Nature, 2008, 453, 499-504.

3 V. Gopalakrishnan and C. F. Zukoski, J. Rheol., 2007, 51, 623-644.

4 T. Gibaud, D. Frelat and S. Manneville, Soft Matter, 2010, 6, 3482-3488.

5 Colloidal drug delivery systems, ed. J. Kreuter, Marcel Dekker Inc., NY, 1994, vol. 66.

6 Food polymers, gels and colloids, ed. E. Dickinson, Woodhead Publishing Ltd, Cambridge, UK, 1991.

7 A. S. Michaels and J. C. Bolger, Ind. Eng. Chem. Fund., 1962, 1, 24-33.

8 J.-M. Condre, C. Ligoure and L. Cipelletti, J. Stat. Mech., 2007, P02010.

9 R. Buscall, T. H. Choudhury, M. A. Faers, J. W. Goodwin, P. A. Luckhamb and S. J. Partridge, Soft Matter, 2009, 5, 1345-1349.

10 J. Sprakel, S. B. Lindström, T. E. Kodger and D. A. Weitz, Phys. Rev. Lett., 2011, 106, 248303.

11 D. Bonn, H. Kellay, M. Prochnow, K. Ben-Djemiaa and J. Meunier, Science, 1998, 280, 265-267.

12 P. J. Skrzeszewska, J. Sprakel, F. A. de Wolf, R. Fokkink, M. A. Cohen Stuart and J. van der Gucht, Macromolecules, 2010, 43, 3542-3548.

13 J. C. Conrad, H. M. Wyss, V. Trappe, S. Manley, K. Miyazaki, L. J. Kaufman, A. B. Schofield, D. R. Reichman and D. A. Weitz, J. Rheol., 2010, $\mathbf{5 4}, 421-438$

14 H. Hoekstra, J. Vermant, J. Mewis and G. G. Fuller, Langmuir, 2003, 19, 9134-9141.

15 C. O. Osuji, C. Kim and D. A. Weitz, Phys. Rev. E, 2008, 77, 060402.

16

17 A. Zaccone, H. Wu and E. Del Gado, Phys. Rev. Lett., 2009, 103, 208301.

18 H. A. Kramers, Physica, 1940, 7, 284-304.

19 E. Evans and K. Ritchie, Biophys. J., 1997, 72, 1541-1555.

20 G. I. Bell, Science, 1978, 200, 618-627.

21 E. Evans, Аnпи. Rev. Biophys. Biomolec. Struct., 2001, 30, 105-128.

22 P. M. Williams, Analytica Chimica Acta, 2003, 479, 107-115.

23 Handbook of mathematical functions, ed. M. Abramowitz and I. A. Stegun, National Bureau of Standards, 1965, pp. 228-229.

24 A. Garcímartin, A. Guarino, L. Bellon and S. Ciliberto, Phys. Rev. Lett., 1997, 79, 3202-3205.

25 S. Mahesh and S. L. Phoenix, Int. J. Fract., 2004, 127, 303-360.

26 W. Weibull, Appl. Mech. Rev., 1952, 5, 449-451.

27 H. Tanaka and T. Araki, EPL-Europhys. Lett., 2007, 79, 58003.

28 A. Zaccone, H. Wu, D. Gentili and M. Morbidelli, Phys. Rev. E, 2009, 051404.

29 H. H. Johnson, J. G. Morlet and A. R. Troiano, T. Am. I. Min. Met. Eng., 1958, 212, 528-536.

30 M. Chaplain and G. Valentin, Eur. J. Wood Wood Prod., 2007, 65, 7-16.

31 R. W. Davidge, J. R. McLaren and G. Tappin, J. Mater. Sci., 1973, 8, 1699-1705. 